\title{
An Equivalent Circuit Model of a Commercial LED with an ESD Protection Component for VLC
}

\author{
Xicong Li, Zabih Ghassemlooy, Senior Member, IEEE, Fellow, OSA, Stanislav Zvanovec, Senior Member, IEEE, \\ and Luis Nero Alves, Member, IEEE
}

\begin{abstract}
An equivalent circuit model for a typical commercial light-emitting diode (LED) used in visible light communication (VLC) applications is proposed. The parameters of the elements in the model are extracted from the measured impedance using a vector network analyser. The model shows high accuracy in fitting the measured results and shows the effect of bonding/packaging parasitics and the electrostatic discharge (ESD) component on the LED's impedance and bandwidth. This study aims to raise the designer's awareness when designing and fabricating high-speed LED/ $\mu$ LED devices to achieve the optimal modulation bandwidth in the emerging VLC applications.
\end{abstract}

Index Terms-VLC, LiFi, LED, impedance, equivalent circuit model, ESD, parasitics

\section{INTRODUCTION}

$\mathbf{V}$ ISIBLE light communication (VLC)) or light fidelity (LiFi) has evolved from Mbps to Gbps in terms of the data rates with international standards supported over the past decade [1]. Such high-speed communication requires modulating the light intensity of light-emitting diodes (LED) or laser diodes (LD) in a wide bandwidth, imposing a high demand on the optimised fabrication process to deliver faster devices, like $\mu$ LEDs [2] and white lasers, for VLC applications. However, the majority of VLC/LiFi products or laboratory-based systems will have to rely on off-the-shelf devices, especially the high-power LEDs intended for illumination, for the time being and possibly for a long time. Additionally, most research works reported on high-bandwidth LED/ $\mu$ LED designs have been carried out by probing the chip die directly or using short bonding wires without complete packaging or electrostatic discharge (ESD) protection. Therefore, characterising the LEDs widely adopted in current VLC systems using the ac smallsignal equivalent circuit model has great significances in: (i) providing an accurate model for the LED driver/modulator design; and (ii) revealing the parasitics and their effects to provide guidance for future wideband device design.

In this letter, we propose an extended equivalent circuit model for a typical commercial phosphor-converted white LED, which includes parasitics introduced by the bonding

This work was supported by the COST Action NEWFOCUS CA19111 and the H2020 under the Marie Sklodowska-Curie grant agreement no 764461 (VisIoN). (Corresponding author: Xicong Li.)

X. Li and Z. Ghassemlooy are with Optical Communications Research Group (OCRG), Faculty of Engineering and Environment, Northumbria University, NE1 8ST, Newcastle, UK (e-mail: xicong.li@ northumbria.ac.uk).

S. Zvanovec is with Department of Electromagnetic Field, Faculty of Electrical Engineering, Czech Technical University, Prague, Czech Republic.

L. Alves is with Department of Electronics Telecommunications and Informatics, University of Aveiro, Aveiro, Portugal.

Manuscript received XX XX, XXXX; revised XX XX, XXXX.

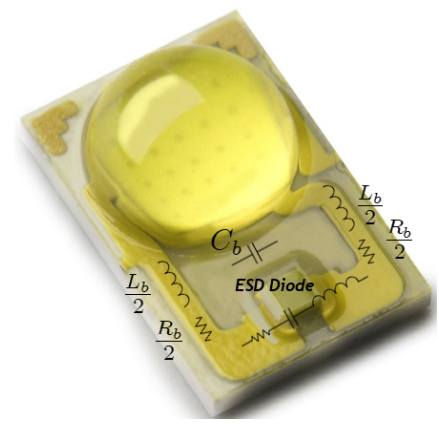

(a)

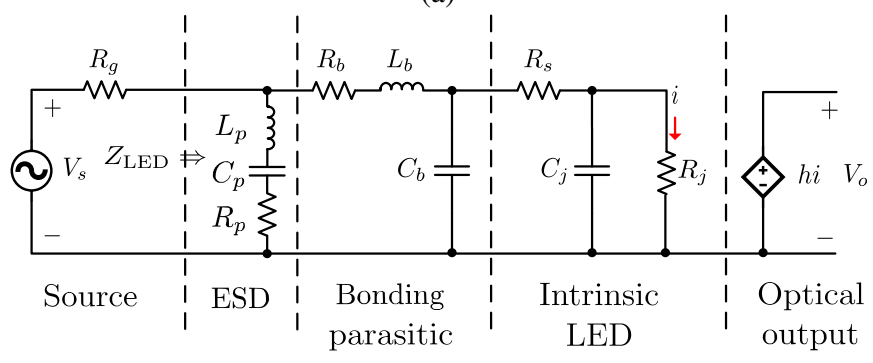

(b)

Fig. 1: (a) A photo of the commercial LED in use; and (b) the equivalent circuit model of the LED driven by a source with an output impedance $R_{g}=50 \Omega$.

wires/tracks and ESD protection components compared with the classical model [3]. The equivalent circuit parameters are extracted from the impedance measured by a vector network analyser (VNA) with a dedicated surface-mount device (SMD) test fixture. Good agreement between the measured impedance and the extracted model is achieved. With the model, we observe that two resonance peaks are incurred at high frequencies due to the bonding wire parasitics and the ESD component, respectively. Although current commercial LEDs can rarely be modulated at that high speed, the observation raises awareness about the negative effects of parasitics introduced by bonding, packaging, and ESD components in the high-speed LED/ $\mu \mathrm{LED}$ design specifically tailored for VLC applications [4], [5].

\section{THEORY}

The device used in our investigation is an off-the-shelf phosphor-converted white LED (Lumileds Luxeon Rebel LXML-PWC2) with a system PCB packaging. Fig. 1(a) shows the structure of the device in use. It can be seen that, in parallel with an ESD diode, the LED die is soldered on a compact PCB 
with bonding wires/tracks running from the top layer to the bottom layer to connect to the pads. Fig. 1(b) schematically illustrates the corresponding equivalent circuit model.

Both our previous study [6] and the works reported in the literature [7] have shown that, despite the difference in the LED packaging, the intrinsic LED model, i.e., the classical model, can fit the measurement well with good accuracy in the low-frequency range where the parasitics are not dominant. As shown in Fig. 1(b), the intrinsic LED part consists of the junction capacitor $C_{j}$, the junction dynamic resistance $R_{j}$, and the serial resistance $R_{s}$ accounting for the parasitic inside the LED die. Note that the current $i$ through $R_{j}$ reflects the effective current for the intensity modulation. The second part of the circuit model represents the bonding wire/track parasitics with the serial resistance $R_{b}$, the serial inductance $L_{b}$, and the capacitor $C_{b}$ in parallel with the intrinsic LED model. As shown later, $L_{b}$ is the dominant component in the mid-range frequencies. To model the ESD diode, a serial $R L C$ (i.e., a combination of $R_{p}, L_{p}$, and $C_{p}$ ) circuit is included [8]. It is worth mentioning that the ESD component is critical in protecting the LED/LD die from electrostatic discharge damage and therefore is highly essential to be integrated into mass-produced LED/LD chips. In this work, the device under investigation uses a separate Zener diode for ESD protection, while many LED devices might implement it within the die [9]. It will be shown that the third $R L C$ circuit will dominate the response beyond its resonance frequency. It is worth noting that, for small-size ESD components or the ESD circuitry in the die, the $R L C$ parasitic elements can be neglectable in the measured frequency range. Due to the introduction of the $R L C$ circuit, the proposed model can cover a wider range of LEDs, which has been experimentally validated on more than six different LEDs with different sizes and packaging.

The analytical expression of the LED's impedance can therefore be written as:

$$
\begin{aligned}
Z_{\mathrm{LED}}= & \underbrace{\left[\left(R_{s}+\frac{1}{j \omega C_{j}} / / R_{j}\right)\right.}_{\text {intrinsic model }} / / \underbrace{\left.\frac{1}{j \omega C_{b}}+R_{b}+j \omega L_{b}\right]}_{\text {bonding parasitic }} \\
& / / \underbrace{\left[R_{p}+\frac{1}{j \omega C_{p}}+j \omega L_{p}\right]}_{\text {ESD parasitic }},
\end{aligned}
$$

where $j$ is the imaginary unit, $\omega$ is the angular frequency, and $/ /$ denotes two impedances in parallel. Using the voltage $V_{R_{j}}$ over $R_{j}$ to proportionally represent the current for intensity modulation [10], the frequency response of the LED can be predicted by the following transfer function:

$$
H(j \omega)=\frac{V_{o}}{V_{s}}=\frac{h i}{V_{s}}=\frac{h}{R_{j}} \frac{V_{R_{j}}}{V_{s}},
$$

where $h$ is the gain coefficient accounting for the photodetector's responsitivity and the gain introduced by subsequent circuitry, e.g., transimpedance amplifier.

\section{MEASUREMENT AND PARAMETER EXTRACTION}

We measured the LED's impedance using a VNA (Keysight E5061B, 5Hz-3GHz) and its wideband bottom electrode SMD

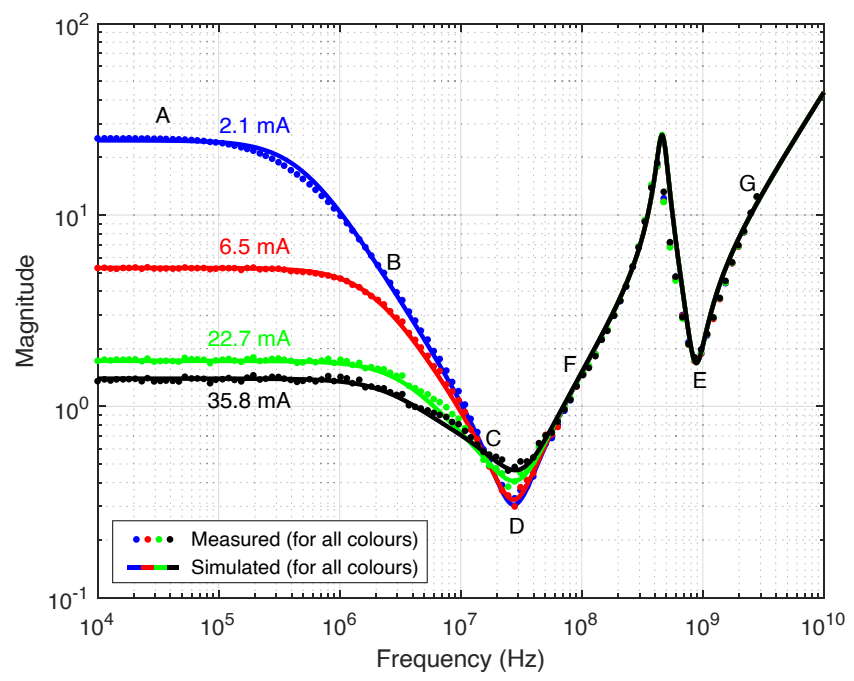

(a)

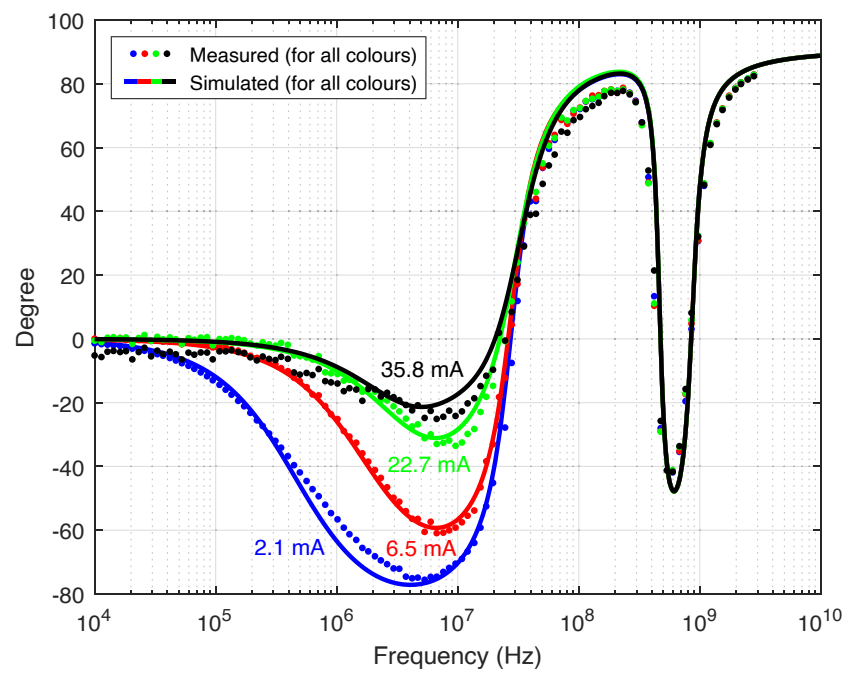

(b)

Fig. 2: Measured and simulated: (a) $\left|Z_{\mathrm{LED}}\right|$, and (b) $\angle Z_{\mathrm{LED}}$.

test fixture (Keysight 16197A, 3GHz) at varying bias points. The LED mounted on the fixture was directly powered by the VNA's built-in bias-tee to avoid additional parasitics. Using the first reflection port, which connects to the LED, the impedance can be measured based on the S11 parameter. The second port of the VNA was connected to a high-speed optical receiver (Newport 1601, 30kHz-1GHz) to measure the $\mathrm{S} 21$ parameter for comparison with the model-predicted frequency response. All the measurements were carried out following a full calibration with fixture de-embedding was conducted to the VNA. Since the VNA is optimised with an impedance measurement function, the complex-valued impedance can be directly measured without conversion from the S11 parameter.

Fig. 2(a) shows the measured and simulated impedance magnitude $\left|Z_{\mathrm{LED}}\right|$ using the extracted parameters given in Table I for a range of bias currents. The parameter extraction method is briefly described as follows. In the low-frequency range as marked by zone $\mathrm{A}$ in Fig. 2(a), the measured 
impedance is used to estimate $\left(R_{j}+R_{s}+R_{b}\right)$. The following decaying slopes in zones $\mathrm{B}$ and $\mathrm{C}$ are used to determine $C_{j}$ and $C_{b}$, respectively. The minimum impedance points of $\mathrm{D}$ and $\mathrm{E}$ correspond to $R_{b}$ and $R_{p}$, whereas $L_{b}$ and $L_{p}$ are estimated using the slopes in zones F and G. Additionally, the resonance frequencies, which can be clearly distinguished in zones $\mathrm{D}$ and $\mathrm{E}$, confirm or estimate the value of $C_{b}$ and $C_{p}$. Then the other parameters can be determined using the least-square error fitting. Since the parameters were extracted using measured $\left|Z_{\mathrm{LED}}\right|$ only, the match between the measured and simulated impedance phase $\angle Z_{\text {LED }}$ confirms the validity of the proposed model, see Fig. 2(b).

TABLE I: Extracted Parameters at Varying Bias Currents

\begin{tabular}{|c|c|c|c|c|}
\hline \multirow{2}{*}{ Item } & \multicolumn{4}{|c|}{ Bias current $(\mathrm{mA})$} \\
\cline { 2 - 5 } & $2.1 \mathrm{~mA}$ & $6.5 \mathrm{~mA}$ & $22.7 \mathrm{~mA}$ & $35.8 \mathrm{~mA}$ \\
\hline$R_{j}(\Omega)$ & 21.35 & 2.51 & 0.74 & 0.50 \\
\hline$C_{j}(\mathrm{nF})$ & 0.98 & 8.70 & 38 & 80.0 \\
\hline$R_{s}(\Omega)$ & 2.93 & 2.51 & 0.74 & 0.62 \\
\hline$R_{b}(\Omega)$ & 0.29 & 0.27 & 0.25 & 0.28 \\
\hline$C_{b}(\mathrm{nF})$ & 13.2 & 14.4 & 14.4 & 14.4 \\
\hline$L_{b}(\mathrm{nH})$ & 2.45 & 2.45 & 2.45 & 2.45 \\
\hline$R_{p}(\Omega)$ & 1.72 & 1.72 & 1.72 & 1.72 \\
\hline$C_{p}(\mathrm{pF})$ & 34.4 & 34.4 & 34.4 & 34.4 \\
\hline$L_{p}(\mathrm{nH})$ & 0.98 & 0.98 & 0.98 & 0.98 \\
\hline \multicolumn{5}{|r}{}
\end{tabular}

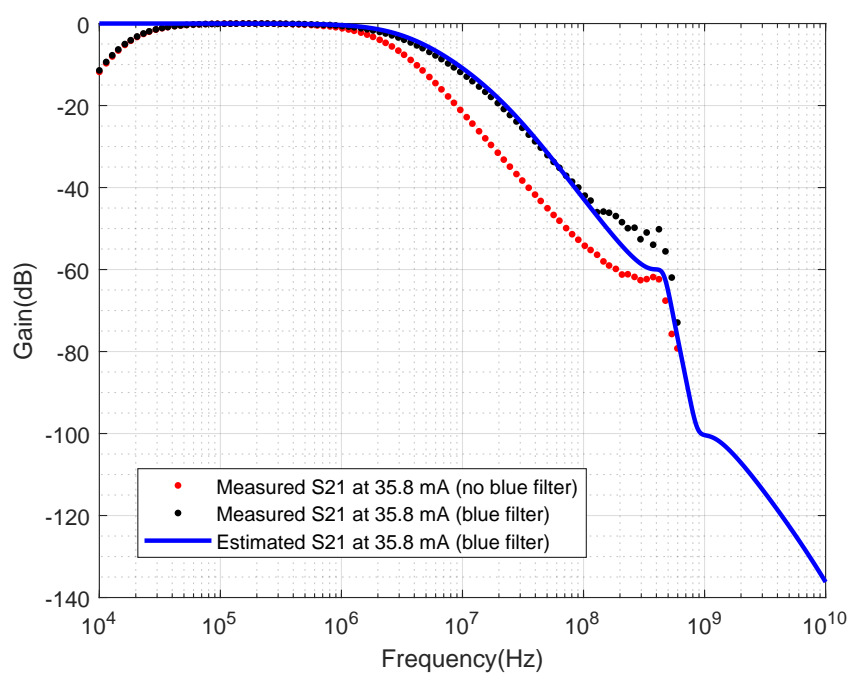

Fig. 3: Measured and simulated S21 with and without the blue filter.

Using (2) with the extracted parameters, the LED's frequency response was predicted and plotted in Fig. 3. Also shown is the measured S21 parameter after normalisation being applied. Due to the coated phosphor which converts part of the blue spectrum to longer wavelengths to produce white light for illumination, we used a blue filter to remove the slow components generated by the phosphor to obtain the real frequency response of the LED [11]. Fig. 3 shows a good agreement between the model-predicted and the measured S21 parameter curve at the bias current of $35.8 \mathrm{~mA}$, confirming the effectiveness of our model. Here, a comparison between the model prediction and measurement was only given at the highest bias current because the blue filter and the limited gain of the receiver cannot support accurate S21 measurement at low bias currents. From the measurements and extracted parameters, it can be observed that the 3-dB bandwidth of the LED under test is mainly limited by the junction capacitance and the dynamic resistance [5], which can be roughly estimated by $f_{3 d B}=1 / 2 \pi R_{j} C_{j}$. As shown in Fig. 3 and confirmed by the simulated model, the bonding parasitics introduce steeper attenuation slopes in the frequency range of $50 \sim 100 \mathrm{MHz}$, while the ESD diode introduces an additional degree of steepness to the slopes beyond $500 \mathrm{MHz}$. Note that the knee frequencies depend on the ESD parasitics and vary from device to device.

\section{CONCLUSion}

We proposed a complete equivalent circuit model of an offthe-shelf LED used in VLC systems, taking into account the LED's intrinsic model and parasitics introduced by the die bonding/packaging and the ESD component. With this model and the measured impedance, parameters were extracted and we showed that there is a good agreement between the measured and the simulated results. Furthermore, we showed that the predicted frequency response matched the measured S21parameter, indicating that the bonding parasitics prevent the LED from achieving high bandwidth in the middle-frequency range while the ESD diode introduced steep attenuation slopes in the high-frequency range. The proposed method based on the equivalent circuit model and S-parameters measurement can be applicable to characterising future high-speed LED/ $\mu$ LED devices customised for VLC systems. Finally, experimental investigation demonstrated the effectiveness of the proposed method and provided a set of real-world data for future references.

\section{REFERENCES}

[1] Z. Ghassemlooy, L. N. Alves, S. Zvanovec, and M.-A. Khalighi, Visible Light Communications: Theory and Applications. CRC Press, 2017.

[2] R. X. G. Ferreira, E. Xie, J. J. D. McKendry, S. Rajbhandari, H. Chun, G. Faulkner, S. Watson, A. E. Kelly, E. Gu, R. V. Penty, I. H. White, D. C. O'Brien, and M. D. Dawson, "High bandwidth GaN-based microleds for multi-Gb/s visible light communications," IEEE Photonics Technology Letters, vol. 28, no. 19, pp. 2023-2026, 2016.

[3] T. P. Lee, "Effect of junction capacitance on the rise time of LED's and on the turn-on delay of injection lasers," The Bell System Technical Journal, vol. 54, no. 1, pp. 53-68, 1975.

[4] L. Maret, A. Lagrange, and L. Dupré, Ultra-high speed optical wireless communications with gallium-nitride microLED, ser. SPIE OPTO. SPIE, 2021, vol. 11706.

[5] R. Sujan, J. D. M. Jonathan, H. Johannes, C. Hyunchae, F. Grahame, H. Harald, M. W. Ian, O. Dominic, and D. D. Martin, "A review of gallium nitride LEDs for multi-gigabit-per-second visible light data communications," Semiconductor Science and Technology, vol. 32, no. 2, p. 023001, 2017.

[6] X. Li, Z. Ghassemlooy, S. Zvanovec, M. Zhang, and A. Burton, "Equivalent circuit model of high power LEDs for VLC systems," in 2019 2nd West Asian Colloquium on Optical Wireless Communications (WACOWC), Conference Proceedings, pp. 90-95.

[7] J. P. M. G. Linnartz, X. Deng, A. Alexeev, and S. Mardanikorani, "Wireless communication over an led channel," IEEE Communications Magazine, vol. 58, no. 12, pp. 77-82, 2020.

[8] Y. Huang, C. Lin, and T. Wu, "A simple method to improve signal integrity of electrostatic discharge protection devices," in 2017 IEEE 26th Conference on Electrical Performance of Electronic Packaging and Systems (EPEPS), Conference Proceedings, pp. 1-3.

[9] S. J. Chang, C. H. Chen, Y. K. Su, J. K. Sheu, W. C. Lai, J. M. Tsai, C. H. Liu, and S. C. Chen, "Improved ESD protection by combining InGaN-GaN MQW LEDs with GaN Schottky diodes," IEEE Electron Device Letters, vol. 24, no. 3, pp. 129-131, 2003. 
[10] R. S. Tucker and D. J. Pope, "Microwave circuit models of semiconductor injection lasers," IEEE Transactions on Microwave Theory and Techniques, vol. 31, no. 3, pp. 289-294, 1983.

[11] G. Stepniak, M. Schüppert, and C. Bunge, "Advanced modulation formats in phosphorous LED VLC links and the impact of blue filtering," Journal of Lightwave Technology, vol. 33, no. 21, pp. 4413-4423, 2015. 